Indian J Anim Health (2021), 60(1) : 90-97

DOI: 10.36062/ijah.60.1.2021.90-97

\title{
Molecular characterization and phylogenetic analysis of canine parvovirus strains in Andhra Pradesh, India
}

\author{
G. Deepika Kumari ${ }^{1 *}$, R. N. Ramani Pushpa ${ }^{2}$, K. V. Subramanyam ${ }^{3}$, T. Srinivas Rao ${ }^{4}$, \\ K. Satheesh ${ }^{5}$ and V. M. Vivek Srinivas ${ }^{6}$
}

${ }^{1}$ Buffalo Research station, Venkataramannagudem- 534 006, Andhra Pradesh, India; ${ }^{2}$ Department of Veterinary Microbiology, NTR College of Veterinary Science, Gannavaram- 521 102, Andhra Pradesh, India; ${ }^{3}$ Krishi Vigyan Kendra, Guntur- 522 034, Andhra Pradesh, India; ${ }^{4}$ Department of Veterinary Public Health and Epidemiology, NTR College of Veterinary Science, Gannavaram- 521 102, Andhra Pradesh, India; ${ }^{5}$ Animal Husbandry Polytechnic, Banvasi, Kurnool- 581 318, Andhra Pradesh, India; ${ }^{6}$ Rajiv Gandhi Institute of Veterinary Education and Research, Puducherry- 605 009, India

\begin{abstract}
The present study was aimed to characterize the circulating strains isolated from different districts of Andhra Pradesh by phylogenetic analysis and sequencing of the highly variable VP2 (viral protein) capsid protein gene of canine parvovirus (CPV). Phylogenetic analysis was done on randomly selected 18 PCR-positive samples from a collection of 342 faecal samples suspected of CPV. A commercially available canine parvovirus vaccine strain was also included in the study. Of the 19 samples sequenced, the vaccine was of CPV-2 and of the remaining 18 sequenced samples, 13 were new CPV-2a, 4 were new CPV-2b variants and one sample might be a further mutated variant of new CPV-2a. The CPV isolates were having $\mathbf{9 9 . 3 5}$ to $100 \%$ similarity with the CPV strains of other countries. The circulating strains also had a similarity of $99.99 \%$ to $100 \%$ with reference strains present in the GenBank. The phylogenetic analysis revealed that the virus strains formed a close ancestral relationship with the Indian and Chinese strains of CPV-2a and CPV-2b. It could be concluded that new CPV-2a was found to be the predominant strain circulating in Andhra Pradesh besides the presence of a few new CPV-2b and a further mutated variant of new CPV-2a at the adjacent borders of other states with Andhra Pradesh.
\end{abstract}

Key words: Canine parvovirus (CPV), Phylogenetic analysis, VP2 gene sequencing

Canine parvovirus-2 (CPV-2) is responsible for the enteric viral infections in canine and feline. Evolutionary changes in viruses arise due to antigenic shift or antigenic drift in the viral genome, finally conforming new variants of the virus. Mutations in a viral gene were highly suspected in RNA viruses possessing a segmented genome but it was observed rarely in a DNA single stranded virus i.e., CPV. Canine parvovirus has a linear negative sense, genome of $5.2 \mathrm{~kb}$ possessing two open reading frames ORF1 and ORF2. ORF2 encodes $V P 1$ and $V P 2$ proteins of which $V P 2$ is an immune-dominant and relatively unstable protein of the icosahedral symmetry, any substitutions in the amino acid sequence results in the generation of new variants (Kulkarni and Deshpande, 2016). The virus
CPV-2 was first reported in 1978 and was antigenically different from CPV-1, highly contagious and spread worldwide causing high mortality by inducing haemorrhagic gastroenteritis in dogs (Silva et al., 2017). CPV-2 was later altered in at least five or six amino acid positions forming the new variants namely CPV-2a and CPV-2b. Further, additional changes took place at the amino acid position 297 (Ser - Ala) paving to new CPV-2a and new CPV-2b. In the year 2000, another variant with the amino acid substitution at 426 (Asp - Glu) and named as CPV-2c (Buonavoglia et al., 2001). The three fold domain on the capsid protein constituting the amino acids of VP2 426 and 297 are spatially closer to amino acids 97 and 300 and this region throws importance as the transferrin receptor (TFR) of the host which 
interacts and initiates the infection determines host range and highly antigenicity (Allison et al., 2016; Dei Giudici et al., 2017). In the last forty years, CPV-2 has undergone several mutations globally and lead to formation of new variants namely CPV-2a, CPV-2b, CPV-2c, New CPV-2a, New CPV-2b with varying pathogenic potentialities (Fan et al., 2016; Fatima et al., 2017; Nandi et al., 2019) and completely replaced the original CPV-2 prototype and it no longer circulated in canine population (Brindhalakshmi et al., 2016).

The objective of the present study is to analyze the sequences of VP2 gene of CPV present in Andhra Pradesh and their phylogenetic analysis could be useful in identifying the prevalent strain and also to explore any existence of the new variants of $\mathrm{CPV}$ that would be useful in formulating new vaccines to control the disease.

During the present study, a total of 342 rectal swabs were collected from different districts of Andhra Pradesh during the months of Feb 2018 to March 2019 from suspected CPV infected dogs. Out of which only 18 PCRpositive samples and one commercially available vaccine were used for phylogenetic analysis (Table 1). The samples were collected from Super Specialty Veterinary Hospital, Vijayawada, Teaching Veterinary Clinical Complex, NTR College of Veterinary Science, Gannavaram and College of Veterinary Science, Tirupati and from various Veterinary Polyclinics across different districts. The faecal samples were collected in the form of a rectal swab using presterilized swabs containing $0.2 \mathrm{M}$ Sorensons PBS, pH 7.0 neatly labeled and immediately transferred to $-20^{\circ} \mathrm{C}$ till further processing.

One $\mathrm{mL}$ of the emulsified faecal sample was centrifuged at $6,000 \mathrm{rpm}$ for $15 \mathrm{~min}$ at $4^{\circ} \mathrm{C}$. The supernatant was stored at $-80^{\circ} \mathrm{C}$ until it was further used for PCR and virus isolation. The DNA was isolated as per the method described by Vieira et al. (2008). Processed faecal sample

Table 1. Details of samples from different districts for Nucleic acid sequencing with their accession numbers submitted in Gen Bank

\begin{tabular}{clcc}
\hline Sl. No & Place & Designated Name & Accession numbers \\
\hline 1 & Srikakulum & G1 & MT078795 \\
2 & Vijayawada & G2 & MT078796 \\
3 & Vijayawada & G3 & MT078797 \\
4 & Guntur & G4 & MT078798 \\
5 & Guntur & G5 & MT078799 \\
6 & Tirupati & G6 & MT078800 \\
7 & Kadapa & G7 & MT078801 \\
8 & Kadapa & G8 & MT078802 \\
9 & Kadapa & C1 & MT078803 \\
10 & Kurnool & C2 & MT078804 \\
11 & Kurnool & C3 & MT078805 \\
12 & Kurnool & C4 & MT078806 \\
13 & Visakhapatnam & C5 & MT078807 \\
14 & Visakhapatnam & C6 & MT078808 \\
15 & East Godavari & C7 & MT078809 \\
16 & East Godavari & C8 & MT078810 \\
17 & Kurnool & C9 & MT078811 \\
18 & Visakhapatnam & C10 & MT078812 \\
19 & Vaccine & C11 & MT078813 \\
\hline
\end{tabular}


of $100 \mu \mathrm{L}$ was used for template DNA preparation. The samples were boiled at $96^{\circ} \mathrm{C}$ for $10 \mathrm{~min}$ and immediately chilled in crushed ice (Decaro et al., 2005) followed by centrifugation at $12,000 \times g$ for $10 \mathrm{~min}$ at $4^{\circ} \mathrm{C}$. The supernatants were diluted 1:5 in distilled water to reduce residual inhibitors of DNA polymerase activity (Decaro et al., 2006) and used as template for PCR.

$$
\text { Primer pair H-forward }
$$

(CAGGTGATGAATTTGCTACA)/ H-reverse (CATTTGGATAAACTGGTGGT) designed by Buonavoglia to detect the specific amino acids at 297, 300, 305, 375 and 426 in the VP2 capsid protein aiding in differentiating the strains of CPV (Buonavoglia et al., 2001). The H primer amplifies $630 \mathrm{bp}$ fragment of the VP2 gene covering all the critical amino acid positions.

Randomly selected best eighteen amplified PCR products along with one commercially available vaccine (Table 1 ) were sequenced specific to VP2 gene in a ABI 3730 DNA analyser and with Chromas lite software. The obtained sequence specificity, nucleotide and amino acid variations with respect to the VP2 gene sequence of canine parvovirus were determined using BLAST [Basic Local Alignment Search Tool] (http://
blast.ncbi.nlm.nih.gov/Blast.cgi) (Raj et al., 2010). The nucleotide variations of the sequenced samples of $V P 2$ gene were aligned with sequences of standard CPV strains (M38246-FPV,M38245-CPV-2,M24003-CPV2a,EU659121-CPV-2b,FJ222821-CPV-2c) using Clustal W (www.ebi.ac.uk/clustal w) and analysed for any nucleotide variation of CPV $V P 2$ gene at positions 3675, 3685, 3699, 3909 and 4064 from the standard ones (Raj et al., 2010). The phylogenetic analysis studies were carried out using twenty canine parvovirus sequences from various regions of the world available in the GenBank. The sequences were aligned using CLUSTAL W 1.8 program and .aln and .nxs files were generated. The .aln file was converted to .meg file using Mega 4.1 (Tamura et al., 2007) and Neighbor Joining (NJ tree) was constructed (bootstrap replicates $=1000$ ) using Kimura 2 parameter method for pair wise deletion at uniform rates (Raj et al., 2010).

Out of 342 suspected CPV feacal samples, 233 were positive by PCR and randomly 18 samples were selected which produced an amplicon size of $630 \mathrm{bp}$ (Fig. 1). The vaccine strain also reacted with the primers specific to VP2 gene.

The Partial VP2 gene sequences of the

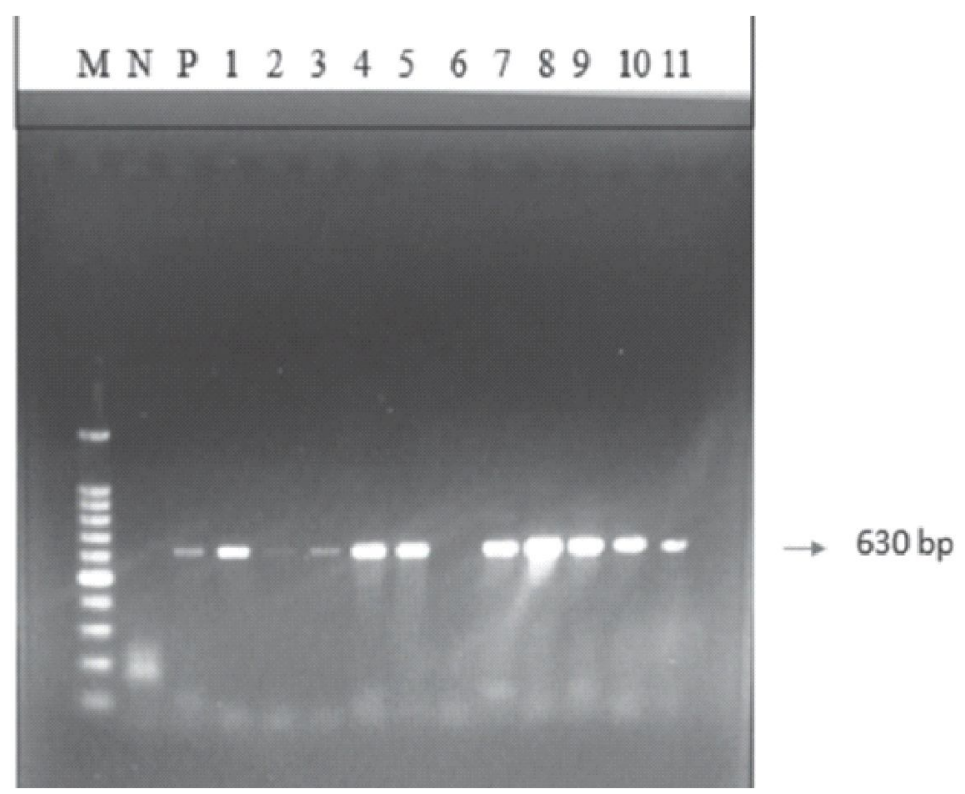

Fig. 1. Polymerase chain reaction of the fecal samples producing an amplicon size of $630 \mathrm{bp}$ VP2 partial gene sequence analysis 
18 samples, along with one vaccine strain were aligned using Mega 7.0 and was compared with the standard reference strains of CPV (CPV-2, New CPV-2a, New CPV-2b and CPV-2c) for the detection of change in amino acids at positions $297,300,305,375$ and 426 , respectively. Out of 18 sequenced samples, 14 were new variant $\mathrm{CPV}-2 \mathrm{a}, 4$ were new variant $\mathrm{CPV}-2 \mathrm{~b}$ and the vaccine strain belonged to prototype CPV-2 (Fig.2).

Fourteen $(\mathrm{C} 1, \mathrm{C} 3, \mathrm{C} 5, \mathrm{C} 6, \mathrm{C} 7, \mathrm{C} 8, \mathrm{G} 1$, G2,G3,G4,G5,G6,G7,G8) sequences had the amino acids Alanine (Ala), Glycine (Gly), Tyrosine (Tyr), Aspartic acid (Asp) and Asparagine (Asn) at residues 297, 300, 305, 375 and 426, respectively. These nucleotide variations were characteristic of new variant CPV-2a. Four $(\mathrm{C} 2, \mathrm{C} 4, \mathrm{C} 9, \mathrm{C} 10)$ had the amino acids Alanine (Ala), Glycine (Gly), Tyrosine (Tyr), Aspartic acid (Asp) and Aspartic acid (Asp) at residues 297, $300,305,375$ and 426, respectively. These nucleotide variations were categorised as new CPV-2b. The vaccine strain (C11) was also sequenced with the amino acids Serine (Ser), Alanine (Ala), Aspartic acid (Asp), Asparagine (Asn) and Asparagine (Asn) at 297, 300, 305, 375 and 426 positions, respectively (Fig. 2).

Phylogenetic analysis of 18 nucleotide sequences of CPV obtained in the present study along with the vaccine strain were used for phylogenetic tree construction with MEGA 4.1 and other twenty reference CPV strains from various parts of the world using neighbour joining method with bootstrap consensus tree (Fig. 3).

The phylogenetic tree was subdivided into two main phylogenetic groups (I and II, boot strap support of 55\%). Group I comprised of 3 clades with CPV-2a in Clade 1 and 3, CPV-2b in Clade 2 and vaccine strain in clade 4 along with the prototype CPV 2 (M38245).

Polymerase chain reaction technique was known for its increased usage as a diagnostic tool for the detection and had been a rapid, sensitive and accurate test for the confirmation of CPV infection (Nandi and

\begin{tabular}{|c|c|c|c|c|c|c|}
\hline Type & 297 аa & 300 aа & 305 aа & 375 aа & 426 aа & Strain \\
\hline CPV-2 & Ser/ TCT & Ala / GCT & Asp / GAT & Asn / AAT & Asn / AAT & Vactine Strain \\
\hline New CPV-2a & $\mathrm{Ala} / \mathrm{GCT}$ & Gly / GGT & TYT/TAT & Aip/GAT & Asn/ AAT & Reference Strain \\
\hline New CPV.2b & $\mathrm{Als} / \mathrm{GCT}$ & Gly/GGT & TV/TAT & Asp/GAT & Asp/ GAT & Reference Strain \\
\hline CPV.2c & $\mathrm{Ala} / \mathrm{GCT}$ & Gly/GGT & Tyr/TAT & Asp/GAT & Glu/GAA & Reference Strain \\
\hline $\mathrm{Cl}$ & Ala / GCI & Gly/GGT & TVI / TAT & Asp/GAT & Asn/ AMT & CPV.2a \\
\hline$C 2$ & $\mathrm{Ala} / \mathrm{GCT}$ & Gly / GGT & Trr/TAT & Asp/GAT & Âsp/GAT & CPV.2b \\
\hline $\mathrm{C}$ & Ala /GCT & Gly/GGT & Trr / TAT & Asp/GAT & Asn/AAT & CPV-2a \\
\hline \begin{tabular}{|c|}
$\mathrm{C}$ \\
\end{tabular} & $\mathrm{Ala}_{2} / \mathrm{GCT}$ & Gly/GGT & Tyr/TAT & Asp/GAT & Asp/ GAT & CPV-2b \\
\hline CS & $\mathrm{Ala} / \mathrm{GCT}$ & Gly/GGI & TVr / TAT & Asip/ GAT & Asn/AMT & CPV.2a \\
\hline C6 & Ala/GCT & Gly/GGT & Trr / TAT & Asp/GAT & Asn/AAT & $C P V \cdot 2 a$ \\
\hline C7 & Ala / GCT & Gly/GGT & TVr/TAT & Asp/GAT & Asn/AAT & CPV-2a \\
\hline $\mathrm{CB}$ & Ala / GCT & Gly / GGT & Trr / TAT & Asp/GAT & Ain/AMT & CPV $2 a$ \\
\hline 9 & $\mathrm{Ala} / \mathrm{GCT}$ & Gy/GGT & Trr/TAT & Asp/GAT & Asp/GAT & CPV.2b \\
\hline C10 & $\mathrm{Ala} / \mathrm{GCT}$ & Gly/GGT & $\mathrm{TV} / \mathrm{TAT}$ & Asp/GAT & Asp/ GAT & CPV-2b \\
\hline C11 & Ser / TCT & $\mathrm{Aa} / \mathrm{GCT}$ & Asp/GAT & Asn/AMT & Asn/AAT & CPV-2 \\
\hline G1 & Ala / GCT & Gly / GGT & TVt/TAT & Asp/GAT & AnN/AAT & CPV-2a \\
\hline 62 & Ala / GCT & Gly/GGT & Trr/TAT & Asp/GAT & Asn/AAT & CPV.2a \\
\hline G3 & $\mathrm{Ala} / \mathrm{GCT}$ & Gy / GGT & Tyr/TAT & Asp/GAT & Asn/AAT & CPV.23 \\
\hline 64 & Ala / GCT & Gly/GGT & Tyr / TAT & Asp/GAT & Asn/AAT & CPV-2a \\
\hline 65 & $\mathrm{Ala} / \mathrm{GCT}$ & Giv/GGT & Trr/TAT & Asp/GAT & Asn/AAT & CPV-2a \\
\hline G6 & Ala / GCT & Gly/GGT & TVt/TAT & Asp/GAT & Asn/AMT & CPV.2a \\
\hline 67 & $\mathrm{Ala} / \mathrm{GCT}$ & Gly/GGT & Trr/TAT & Asp/GAT & Ain/ AAT & CPV-2a \\
\hline 68 & $\mathrm{Ala} / \mathrm{GCT}$ & Gy/GGT & TYr/TAT & Asp/ GAT & AMn/MAT & CPV.23 \\
\hline
\end{tabular}

Fig. 2. Amino acid variation in the VP2 gene of Circulating CPV isolates 


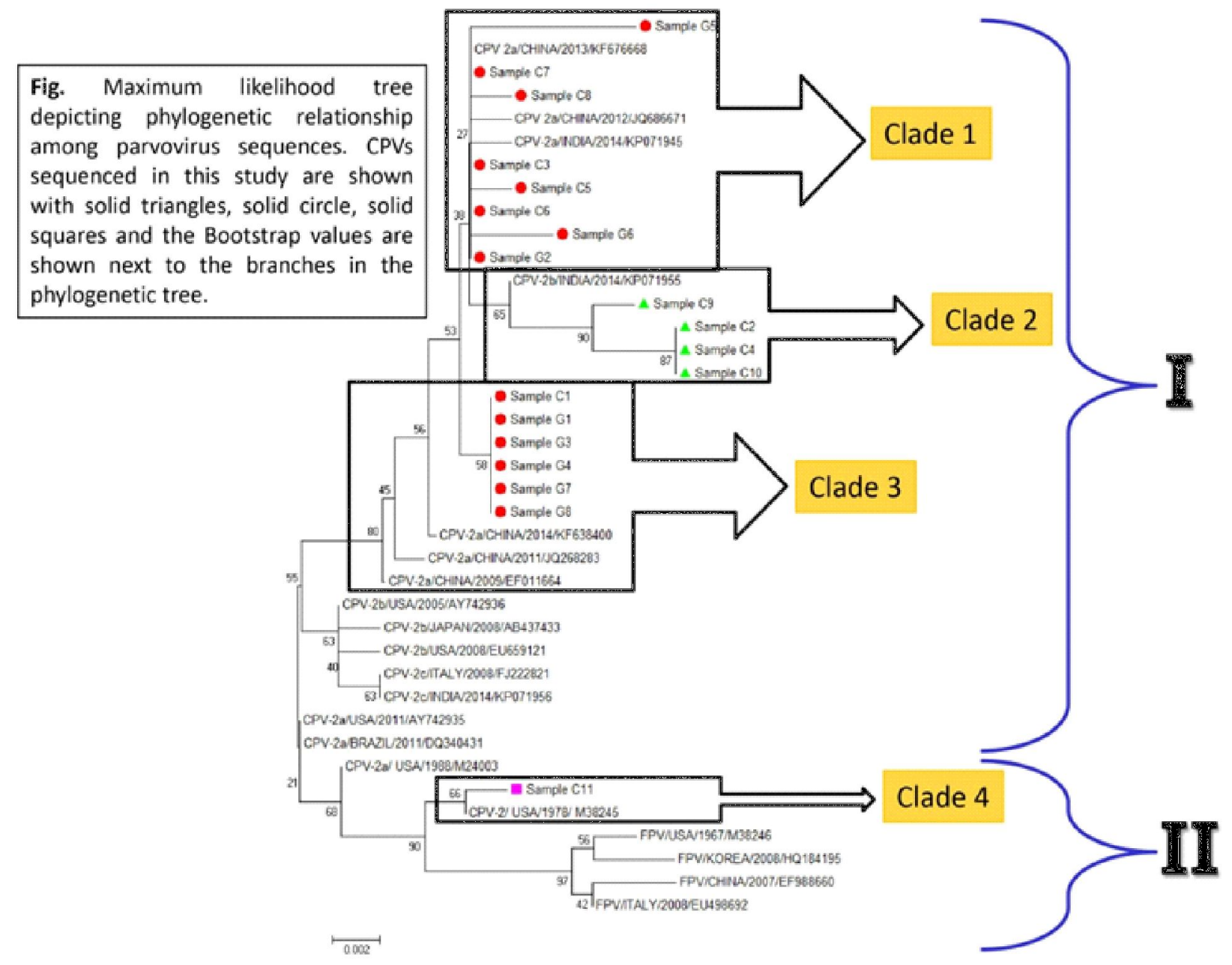

Fig. 3. Phylogenetic analysis of the CPV strains and vaccine strain

Kumar, 2010). In the present study, all 342 fecal samples from clinically ill dogs when screened by PCR, 233 fecal samples were reacted with the primers specific to conserved VP2 region of the genome. Out of 342 samples, 233 samples reacted with VP2 primer pair and recorded an overall prevalence rate of $68.12 \%$ of the canine parvoviral disease in Andhra Pradesh. Srinivas et al. (2013) recorded a prevalence rate of $53.90 \%$ in south India, whereas Agnihotri et al. (2017) reported 36\% of CPV in Haryana from the dogs suspected of viral gastroenteritis using CPV-2ab primers.

The Partial VP2 gene sequences of 18 local CPV isolates along with the vaccine strain was compared with reference strains available in NCBI-GenBank for sequence analysis. The sequences of the 18 isolates were found to be highly specific to CPV and indicated nucleotide identity of $98.35 \%$ to $100 \%$ with VP2 gene sequence of other canine parvovirus reference strains available in the GenBank. The nucleotide sequence identity of the field isolates in the present study was $99.84 \%$ to $100 \%$ with the standard CPV-2a reference strains and $99.35 \%$ with the standard CPV-2b reference strains.

The pair wise distance analysis of VP2 gene of circulating CPV nucleotide sequences of the field isolates when compared with the reference strains was $99.99 \%$ to $100 \%$, and a similar identity was also observed among the field isolates. The nucleotide sequences for local CPV-2a isolates exhibited $99.99 \%$ of homology 
with those of CPV-2b isolates. The nucleotide homology levels between the vaccine strain (CPV-2) and the analysed CPV-2a, CPV-2b isolates were $99.98 \%$ and $99.97 \%$, respectively. The sample (G6) on sequence analysis, the nucleotide sequence identity was $99.66 \%$ with CPV-2a, hence might be a further mutated variant of CPV-2a. Behera et al. (2016) could also detect CPV-2a mutated variant in their study. Sheikh et al. (2017) could find a varying identity of $95-99.5 \%$ from the CPV samples sequenced those were obtained in different locations of Iraq. Srinivas et al. (2013) in their study of characterising the CPV samples of southern India obtained nucleotide homology of 95-99\% which was in accordance with the present study. Zienius et al. (2016) also reported that the Lithuanian strains were showing $99.3 \%$ similarity with the CPV sequences of France, Germany, Italy and Turkey but not identical.

The thirteen new CPV-2a samples and one mutated variant when compared with the standard CPV-2 prototype, they had nucleotide variations at positions $3675(\mathrm{~T}-\mathrm{G}), 3685$ (C G), $3699(\mathrm{G}-\mathrm{T}), 3909$ (A - G) and identical nucleotide $(\mathrm{T})$ at position 4064 , the remaining 4 samples which were new CPV-2b had nucleotide variations at positions $3675(\mathrm{~T}-\mathrm{G})$, 3685 (C - G), 3699 ( G - T ), 3909 (A - G) and at 4064 (A - G). When compared with the CPV2a the thirteen new CPV-2a samples and one mutated variant sequenced had nucleotide variations at position $3675(\mathrm{~T}-\mathrm{G})$. The four new CPV-2b isolates also showed similar nucleotide variations at position $3675(\mathrm{~T}-\mathrm{G})$ with the CPV-2b strain. The commercial available vaccine sequenced was similar at all nucleotide positions with the standard CPV-2 prototype. Similar substitutions in the nucleotide positions of new CPV-2a and CPV-2b were reported in VP2 gene of CPV from Southern India (Srinivas et al., 2013) and Pondicherry (Raj et al., 2010). The G6 sample on nucleotide analysis showed significant change in the sequence and was similar with new CPV-2a sequence but had a variation of $0.18 \%$ with new CPV-2a on BLASTn analysis. The presence of this new mutated variant was detected along with other new CPV-2a strains which suggest that presence and co-occurrence of new mutated variants is possible only with CPV-2a, and not as separate entity (Behera et al., 2016). It might be a completely new variant or a defective viral strain of CPV-2a which needs to be further investigated based on full length sequence analysis.

Four samples (C2, C4, C9, C10) had substitution at 297 (Ser-Ala), 300 (Ala-Gly), 305 (Asp-Try), 375 (Asn-Asp) and 426 (Asn-Asp) from the original CPV-2 and differing from CPV$2 \mathrm{~b}$ at the amino acid position 297 (Ser-Ala), and were characterised as new CPV-2b and no change at 426 Ala-Glu which is unique to CPV2c was not observed in the present study. Similar substitutions in VP2 gene were reported (Raj et al., 2010; Srinivas et al., 2013).

Amino acid substitutions in the VP2 gene sequence could be responsible to affect the antigenic properties of canine parvovirus variants (Truyen et al., 1995).

The partial VP2 nucleotide sequences of circulating CPV along with the other twenty reference CPV strains from different geographical areas available in Genbank were used to construct phylogenetic tree using Neighbor Joining method in MEGA 4.1 with bootstrap consensus. The phylogenetic analysis of CPV-2 variants, CPV-2a and CPV-2b circulating in canine population of Andhra Pradesh fall into two distinct phylogroups and 4 clades. Phylogroup I comprised of clade 1, 2 and 3, whereas phylogroup II comprised of clade 4 . Clade 1 with eight CPV-2a strains (G2,G5,G6,C3,C5,C6,C7,C8) and were having close ancestry relation with the Indian (KP071945) and Chinese (KF676668, JQ686671) CPV-2a strains. Clade 2 comprised of four CPV-2b (C2, C4, C9, C10) and were associated with VP2 sequences from Indian (KP071955) CPV-2b strains, whereas Clade 3 comprised of six CPV-2a (G1, G3, G4, G7, G8, C1) and associated with VP2 sequences of Chinese (KF638400, JQ268283, EF011664) CPV-2a strains. 
The clade 3 comprised of the CPV-2a isolates formed a distinct lineage with the CPV2b strains of Japan (AB437433) and USA (EU659121) suggesting that CPV evolved independently and formed separate lineages. The vaccine strain CPV-2 was closely associated with CPV-2 prototype from USA (M38245) and formed a sub clade with the feline panleukopenia virus of USA (FPV-M38264), China (EF988660), Korea (HQ184195) which was supposed to be ancestor for evolution of CPV and were placed in clade 4 of phylogroup II. Based on phylogenetic analysis the query sequences in the present study were forming a close ancestory relationship with the Indian and Chinese strains of CPV-2a and CPV-2b. In previous reports also the canine parvovirus isolates in India appeared to have evolved independently and formed separate lineages with the South East Asian countries (Chinchkar et al., 2006). The findings of the present study were in accordance with Behera et al. (2016), Thomas et al. (2017) and Zienius et al. (2016).

From the present study it was found that new CPV-2a (Ser- Ala 297) was the dominant strain circulating in Andhra pradesh. New CPV$2 b$ was also seen at the trans-boundaries with

\section{REFERENCES}

Allison AB, Organtini LJ, Zhang S, Hafenstein SL, Holmes EC et al., 2016. Single mutations in the VP2 300 loop region of the three-fold spike of the carnivore parvovirus capsid can determine host range. J Virol, 90(2): 753-767, doi: $10.1128 /$ JVI.02636-15

Agnihotri D, Singh Y, Maan S, Jain VK, Kumar A et al., 2017. Molecular detection and clinicohaematological study of viral gastroenteritis in dogs. Haryana Vet, 56(1): 7276

Behera M, Panda SK, Das S, Sahoo PK, Acharya A et al., 2016. Genomic typing and phylogenetic analysis of canine parvovirus detected in the state of Odisha India. Vet Arch, 86(5): 727-741

Brindhalakshmi B, Mukhopadhyay HK, Antony PX, Thanislass J, Vijayalakshmi P et al., 2016. Isolation and molecular characterization of canine and feline parvovirus strains-an updated other adjacent states. The commercially available vaccine contained only the original CPV-2 prototype but not the other strains. Studies on cross protection of the available vaccine with other strains of CPV should be carried out as vaccinated pups were also being infected by the virus. Continuous studies on canine parvovirus are of significant importance because of its evolutionary genetic changes in the VP2 gene thus generating an idea on the strains that are circulating in Andhra Pradesh. This would be successful to formulate effective control strategies in future for protecting the canine and feline populations.

Conflict of interest: Authors have no conflict of interest in this study.

\section{ACKNOWLEDGEMENTS}

The authors are grateful to Sri Venkateswara Veterinary University, Tirupati for providing the grants to carry out the work. We are also thankful to the Department of Veterinary Microbiology, NTR College of Veterinary Science, Gannavaram for providing the necessary facilities in the research laboratory to perform the work.

review. J Dairy Vet Anim Res, 3(5): 164169, doi: 10.15406/jdvar.2016.03.00093

Buonavoglia C, Martella V, Pratelli A, Tempesta M, Cavalli A et al., 2001. Evidence for evolution of canine parvovirus type 2 in Italy. J GenVirol, 82: 3021-3025, doi: 10.1099/0022-1317-8212-3021

Chinchkar SR, Subramaniam BM, Rao NH, Rangarajan PN, Thiagarajan D et al., 2006. Analysis of VP2 gene of canine parvovirus in India. Arch Virol, 151(9): 1881-1887, doi: 10.1007/s00705-006-0753-8

Decaro N, Elia G, Martella V, Desario C, Campolo M et al., 2005. A real time PCR assay for rapid detection and quantitation of canine parvovirus type 2 DNA in the faeces of dogs. Vet Microbiol, 105(1): 19-28, doi: 10.1016/ j.vetmic.2004.09.018

Decaro N, Martella V, Desario C, Bellacicco AL, 
Camero M et al., 2006. First detection of canine parvovirus type $2 \mathrm{c}$ in pups with haemorrhagic enteritis in Spain. J Vet Med B, 53(10): 468472, doi: 10.1111/j.1439-0450.2006.00974.x

Dei Giudici S, Cubeddu T, Giagu A, Sanna G, Rocca S et al., 2017. First molecular characterization of canine parvovirus strains in Sardinia Italy. Arch Virol, 162(11): 3481-3486, doi: 10.1007/ s00705-017-3457-3

Fan XX, Gao Y, Shu L,Wei YQ, Yao XP et al., 2016. Transcriptome profiling indicating canine parvovirus type $2 \mathrm{a}$ as a potential immune activator. Virus Genes, 52(6): 768-779, doi: 10.1007/s11262-016-1363-5

Fatima U, Mehboob A, Abid M, Khan M, Yaqub T et al., 2017. Molecular characterization and evolutionary analysis of canine parvo viruses in dogs. Hosts and Viruses, 4(2): 34-39

Kulkarni MB and Deshpande AR, 2016. Molecular Characterization and Phylogenetic Analysis of Canine Parvovirus. Thesis submitted to Maharashtra Animal and Fishery Sciences University, Nagpur

Nandi S and Kumar M, 2010. Canine parvovirus: current perspective. Indian J Virol, 21(1): 3144, doi: 10.1007/s13337-010-0007-y

Nandi S, Sharma GK, Gupta V, Deol P, Chander V et al., 2019. Global scenario of canine parvovirus mutants: epidemiology diagnostics and immunoprophylactic agents. JSM Vet Med Res, 2: 12

Raj JM, Mukhopadhyay HK, Thanislass J, Antony PX and Pillai RM, 2010. Isolation, molecular characterization and phylogenetic analysis of canine parvovirus. Infect Genet Evol, 10(8): 1237-1241, dio: 10.1016/j.meegid. 2010.08. 005
Sheikh MO, Rashid PMA, Marouf AS, Raheem ZH, Manjunath S et al., 2017. Molecular typing of canine parvovirus from Sulaimani Iraq and phylogenetic analysis using partial VP2 gene. Bulg J Vet Med, 20(3): 225-235, doi: 10.15547/ bjvm. 1032

Silva SP, Silva LNPP, Rodrigues EDL, Cardoso JF, Tavares FN et al., 2017. Full-length genomic and molecular characterization of canine parvovirus in dogs from north of Brazil. Genet Mol Res, 16(3), doi: 10.4238/gmr16039719

Srinivas VMV, Mukhopadhyay HK, Thanislass J, Antony PX and Pillai RM, 2013. Molecular epidemiology of canine parvovirus in southern India. Vet World, 6(10): 744-749, doi: 10.14202/vetworld.2013.744-749

Tamura K, Dudley J, Nei M and Kumar S, 2007. MEGA4: Molecular Evolutionary Genetics Analysis (MEGA) software version 4.0. Mol Biol Evol, 24(8): 1596-1599, doi: 10.1093/ molbev/msm092

Thomas J, Singh M, Goswami TK and Verma S, 2017. Phylogenetic analysis of the partial VP2 gene of canine parvovirus- 2 from the northern region of India. Vet Arh, 87(1): 57-66

Truyen U and Parrish CR, 1995. The evolution and control of parvovirus host ranges. Sem Virol, 6(5): 311-317, doi: 10.1006/smvy.1995.0037

Vieira MJ, Silva E, Oliveira J, Vieira AL, Decaro N et al., 2008. Canine parvovirus 2c infection in central Portugal. J Vet Diagn Invest, 20(4): 488491, doi: 10.1177/104063870802000412

Zienius D, Lelesius R, Kavaliauskis H, Stankevicius A and Salomskas A, 2016. Phylogenetic characterization of canine parvovirus VP2 partial sequences from symptomatic dogs samples. Pol J Vet Sci, 19 (1): 187-196, doi: 10.1515/pjvs-2016-0023

Received - 20.04.2021, Accepted - 22.05.2021, Published - 01.06.2021

Section Editor: Prof. S. N. Joardar, Associate Editor 